\title{
PCB current identification based on near-field measurements using preconditioning and regularization
}

\author{
Denis Rinas, Patrick Ahl, and Stephan Frei \\ AG Bordsysteme, TU Dortmund University, Dortmund, 44227, Germany \\ Correspondence to: Denis Rinas (denis.rinas@tu-dortmund.de)
}

Received: 14 January 2016 - Revised: 11 June 2016 - Accepted: 21 June 2016 - Published: 28 September 2016

\begin{abstract}
Radiated electromagnetic fields from a PCB can be estimated when the source current distribution is known. From a measured near-field distribution, the PCB source current distribution can be found. Accuracy depends on the measurement method and its limitations, the radiation model and the choice of the observation area. Many known methods are based on optimization algorithms for inverse problems that vary a set of elementary radiation sources and create a radiation model. However, apart from the time-consuming optimization process, such methods find one possible solution for a near-field distribution. As this distribution might not reflect the real current distribution, accuracy outside of near-field scan area can be low. Furthermore numerical problems can often be observed. Solving the given inverse problem with a system of linear equations and complex near-field data it can be very sensitive to noise. Regularization methods and an adjusted preconditioning can increase the accuracy. In this paper, an improved radiation model creation approach based on complex near-field data is presented. This approach is based on regularization methods and extended by current estimations from near-field data. Preconditioning is done considering some physical properties of the PCB and its possible current paths. Accuracy and stability of the method are investigated in the presence of noisy data.
\end{abstract}

\section{Introduction}

Alternative methods for evaluation of electromagnetic emissions from electronic components (e.g. near-field scan methods) have several advantages against antenna measurement methods (e.g. ALSE method from CISPR 25, CISPR 25 Ed.3, 2007). Single field strength values obtained from antenna in transition- or far-field, cannot describe the over- all emission behavior of a device under test (DUT). Reproducibility is often limited (Burghart et al., 2004) and identification of radiating sources can be reached only for simple structures (Nishikata et al., 2014), but obviously not in case of a complex device. The large space requirements and high costs of an antenna measurement environment have to be considered too.

Knowing the fields in an infinite plane above an object means, all information is available to calculate field above this plane (Balanis, 1996). From theoretical point of view this would be sufficient to calculate the far-fields of a printed circuit board (PCB). But there are several problems with such a direct approach, e.g. it is not possible to measure field along an infinite plane, accuracy of measurements is limited and accuracy of near-field to far field transformation can be low. Furthermore obtaining far-fields is often not the only aim, also the identification of radiating and disturbing sources, as mentioned above might be needed. Knowing the sources, strategies for noise reduction can easily be developed. Therefore the inverse problem of the resulting electromagnetic field and the causing current distribution on PCB should be solved.

Near-field scan data based radiation or equivalent current models must be distinguished in two main model types. The first type is the field amplitude-only data based model. Since with increasing frequency measurement errors mainly occur in phase data, the corresponding methods try to retrieve phase information mathematically (Yaccarino and Samii, 1999; Pierri et al., 1999) or try to identify the equivalent currents with neglecting the phase information (Isernia et al., 1996; Regué et al., 2001; Xin et al., 2010a; Rinas et al., 2011; Sijher and Kishk, 2005). These approaches often use optimization algorithms (e.g. search heuristics) in which spatial position, orientation, magnitude and phase of the approxi- 
(a)

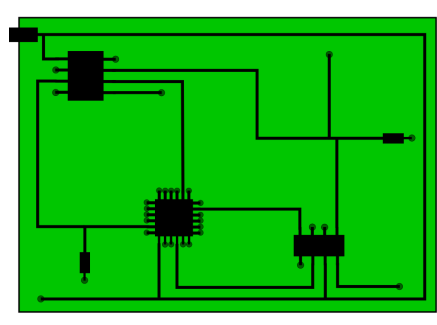

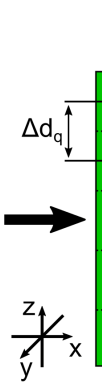

(b)

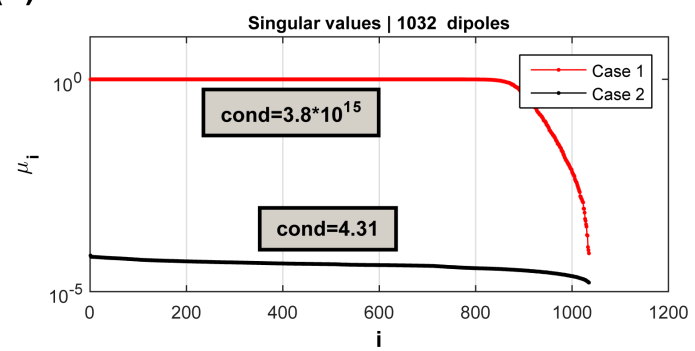

Figure 1. (a) PCB sources approximated by regular grid dipole model. (b) Spectrum $i=[1, N]$ of singular values and condition numbers $\mu_{i}$ for different scan heights and resolution.

mating currents is modified, until the measured near-field distribution of the radiating structure and its model agree. Naturally these approaches can also be applied to complex near-field measurement data. Although these methods often achieve good results, at least in approximating the reference observation plane, the underlying algorithms involve the problem of converging to local minima (Isernia et al., 1996). Besides they can result in a very long computation time. To improve model quality known physical properties of the radiating currents can be included in current distribution estimations. This way the number of free source parameters (Rinas et al., 2011) and sensitivity to noise can be reduced. Resulting radiation pattern is more accurate outside the measured area or volume. Here the trace geometry is assumed to be known, from computer-aided design (CAD) data or the near-field measurements, and the spatial distribution of the possible current paths can be limited. Furthermore current phases are correlated to each other. These improvements lead to reduced computation time and increased accuracy. The second type is the complex field data based model (Laurin et al., 2001; Xin et al., 2010b; Rinas et al., 2013). Here the sources are determined from both magnitude and phase information of the near-field data. Of course optimization algorithms can be applied here again but it is sufficient to solve a system of linear equations using complex data. The set of linear equations can be ill-conditioned and the solution might be erroneous. Regularization techniques can be applied to deal with such problems, e.g. Tikhonov regularization (Xin et al., 2010b; Tichonov and Arsenin, 1977). Furthermore preconditioning of the linear map of the system can be done to create an optimal database for current identification.

In this paper an optimized method for current identification on PCBs based on solving a system of linear equations containing regularization is introduced. Noise sensitivity for current identification process is compared to other methods and need of model preconditioning is shown. Possible current path locations are limited by the locations of the existing traces of the investigated PCBs. Thus there is a benefit due to the physical preconditioning but without the disadvantage of long computation time. This method leads to an equivalent radiation model with good accuracy. Furthermore it can be applied to measurement data obtained with typical EMC test equipment (test receiver, spectrum analyzer) without phase information and therefore an extremely ill-conditioned problem. Improvement of method stability is shown by noise analysis and application to real measurement data.

\section{Current identification method}

To identify currents complex horizontal magnetic near field components $H_{x}$ and $H_{y}$ in a plane above radiating structure are used. As standard and most simple model structure at first equivalent sources are arranged in a grid with resolution $\Delta d_{q}$ above an infinite ground plane (Fig. 1a). Each source consists of a triple of electric dipoles, where each dipole represents a current direction in Cartesian coordinates. The inverse problem between field data and dipole current is solved mathematically with a least squares system and use of pseudoinverse. Following the uniqueness theorem the solution of the inverse problem should produce a good image of the real sources, if the field in a surface around a radiating structure is known. In such a theoretical configuration solving the system of linear equations will create an accurate model with an equivalent current distribution. Obviously the measurement accuracy and size of plane are limited. The radiation model might not be perfect. As result field data will be erroneous or noisy.

\subsection{Noise sensitivity in current identification process}

When solving the inverse problem with a system of linear equations it is important not to amplify noise. To prevent the solution to be very sensitive to noise the problem should not be ill-conditioned. Therefore near-field scan data is analyzed with respect to its variable parameters for preconditioning. To describe the noise amplification problem the onedimensional Fredholm integral equation is used. From Hanson et al. (1971) the solution of Fredholm equation is given 
by

$i_{d}(t)=\sum_{i=1}^{\infty} \frac{\left(u_{i}, h\right)}{\mu_{i}} v_{i}(t)$

Where $i_{d}$ stands for the currents to be identified, $h$ is the known field distribution, $u_{i}$ and $v_{i}$ stand for the singular functions of the Fredholm kernel and $\mu_{i}$ for its singular values. Since $h$ is decomposed to its spectrum it is obvious from Eq. (1) that a small singular value $\mu_{i}$ will strongly amplify the corresponding spectral part of $h$. This means noise, located in the higher frequency region, will be amplified in case of a large spectral condition number (Hanson et al., 1971). In our investigations we used the magnetic field as the known right-hand side values. The currents are reproduced by elementary Hertzian dipoles. The system of linear equations in this case is

$$
\begin{aligned}
& \underbrace{\left[\begin{array}{c}
\underline{\boldsymbol{H}}_{\left(\boldsymbol{r}_{1}\right)} \\
\vdots \\
\underline{\boldsymbol{H}}\left(\boldsymbol{r}_{M}\right)
\end{array}\right]}_{\underline{\boldsymbol{H}}}=\underbrace{\left[\begin{array}{ccc}
\boldsymbol{\Psi}_{1}, \boldsymbol{r}_{Q 1} & \cdots & \underline{\Psi} \boldsymbol{r}_{1}, \boldsymbol{r}_{Q N} \\
\vdots & \ddots & \vdots \\
\Psi \boldsymbol{r}_{M}, \boldsymbol{r}_{Q 1} & \cdots & \underline{\Psi} \boldsymbol{r}_{M}, \boldsymbol{r}_{Q N}
\end{array}\right]}_{\underline{\Psi}} \\
& \quad \times \underbrace{\left[\begin{array}{c}
\underline{\boldsymbol{I}}_{Q 1} \\
\vdots \\
\underline{\boldsymbol{I}}_{Q N}
\end{array}\right]}_{\underline{\underline{\boldsymbol{I}}}}
\end{aligned}
$$

Where vector $\boldsymbol{H}$ contains the known $H$-field values, vector $\boldsymbol{I}$ contains the unknown currents and the matrix $\boldsymbol{\Psi}$ is the linear map containing the geometry and wave propagation. If no further information about sources is available they can be placed for PCBs in form of a regular grid with resolution $\Delta d_{q}$ (Fig. 1a).

To create an accurate radiation model of a PCB the preconditioning of the inverse problems linear map must avoid a high condition number and must allow a wide frequency range. The spectral requirements of the singular values naturally depend on the given structure of the PCB, so that in a strongly restricted singular value spectrum the traces cannot be reproduced accurately. Figure $1 \mathrm{~b}$ shows exemplary the condition numbers and frequency ranges for different nearfield scan linear maps based on simulation data. It can be seen, that for a high scan resolution and high scan height the linear map is bad conditioned. Due to the high number of dipoles the spectrum is wide and the small singular values in high frequencies will strongly amplify the noise (case 1). Whereas in case of a low scan resolution and low scan height the linear map is well conditioned. The low number of dipoles results in a low spectral range (case 2). Of course, the condition number depends on the relation between number of scan points and position of scan points to number of dipoles and position of dipoles. It is not sufficient to reduce scan resolution and scan height and to increase the number of dipoles. Not only that a low number of scan points and a high number of dipoles can cause an under-determined system of equations, but also prevention of undersampling must be ensured (Yaghijan, 1986), following

$\Delta d_{\mathrm{s}}<\lambda /\left(2 \sqrt{1+\left(\lambda / h_{\mathrm{s}}\right)^{2}}\right)$

Where $\lambda$ is the wavelength of the highest frequency, $h_{\mathrm{s}}$ is the height of the scan plane and $\Delta d_{\mathrm{s}}$ stands for the minimal scan resolution.

\subsection{Current estimation of PCB traces using near-field scan data}

Obviously it is not possible to find the best preconditioned linear map for each near-field scan problem. Here regularization methods (e.g. Tikhonov method) can be applied to smoothen the linear system and to generate a less noise sensitive and more accurate model (Xin et al., 2010b; Hanson, 1971). The problem is here given by

$\min \left\{\|A x-b\|_{2}^{2}+\lambda_{t}\left\|\mathbf{L}\left(x-x_{0}\right)\right\|_{2}^{2}\right\}$

Where the parameter $\lambda_{t}$ describes the regularization parameter and $\mathbf{L}$ is a regularization matrix, which can be adapted. In a general approach, the $\mathbf{L}$ matrix is chosen as the identity matrix and $x_{0}$ is set to zero. This can be done when no particular knowledge is available. In Zhenwei et al. (2010) the $\mathbf{L}$ matrix values are extended by a frequency dependency, but without a current estimation for $x_{0}$.

In case of a planar near-field scan above a simple PCB on a ground plane a current estimation from near-field scan can be done. For a single trace, with an assumed infinite length, the current can be approximately calculated from the magnetic field above this trace using image theory (Fig. 2) (IEC/TS 61967-6 Ed.1.0, 2002):

$I_{0 x}=H_{y} \frac{\pi r(r+2 d)}{d}$

With decomposition of $H_{x}$ and $H_{y}$ the current from each trace can be estimated. For high accuracy a sufficiently high scan resolution and low scan height is required. The estimated current amplitudes are implemented in the regularization method and provide an improved convergence to the real current. The problem for the PCB current identification is now given by

$\min \left\{\|\Psi J-H\|_{2}^{2}+\lambda_{t}\left\|\mathbf{I}_{n}\left(I-I_{0}\right)\right\|_{2}^{2}\right\}$

Here the regularization parameter $\lambda_{t}$ contains the Wiener filter as proposed in Hanson (1971) and $\mathbf{I}_{n}$ is the identity matrix.

\subsection{Current path identification}

First we assume the general case that the radiation model consists of equivalent dipoles distributed in a grid above an 


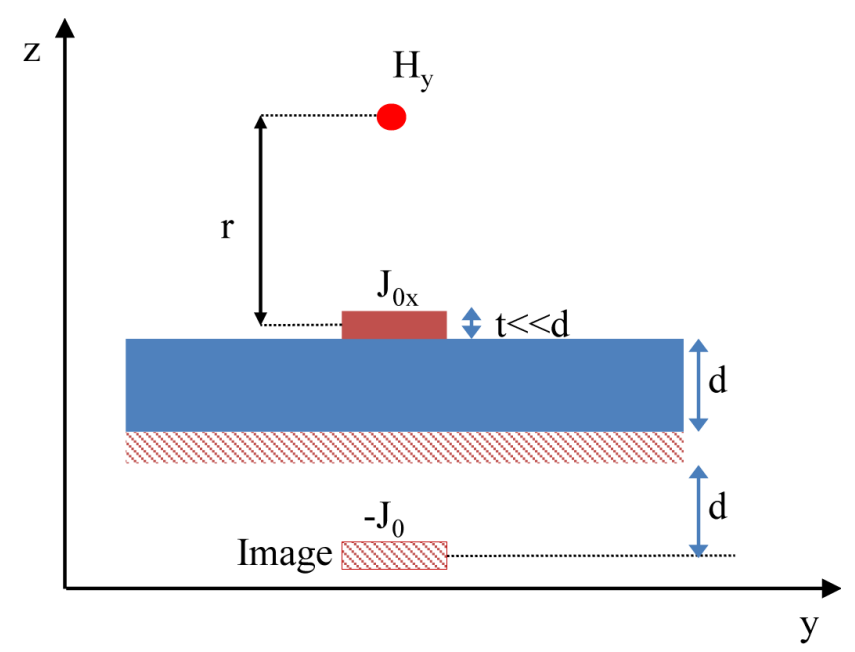

Figure 2. Current estimation from magnetic field of a PCB-trace above ground plane using image theory.

infinite ground plane. As proposed in Sect. 2.2 the amplitude for each current path section can be estimated from the magnetic near-field data. Furthermore this estimation can be used for preconditioning the linear map of the system of linear equations. In a first step the matrix $\boldsymbol{\Psi}$ can be reduced by eliminating the entries from dipoles with a current estimation much smaller than the maximum in its neighborhood. This relation depends on the given signal-to-noise ratio (SNR) and the model resolution.

$\Psi_{n, m}=\left\{\begin{array}{c}0 \text { if } \Psi_{n, m}<\max \left(\underset{\Psi_{n \pm \Delta n, m \pm \Delta m}}{\left(\Psi_{n, m} \text { else }\right.}\right)-(\mathrm{SNR}+\mathrm{s}) \\ \Psi_{n}\end{array}\right.$

Where the index $n+\Delta n$ and $m+\Delta m$ describe the neighborhood and $s$ is a threshold value to be defined. In a second step the orientation of the current path can be used to reduce the dipole triple at each grid point to its decomposed parts following the estimated current path.

Obviously, arrangement of PCB current paths into a regular grid leads to discretization errors (Fig. 3). The approximating dipole path is shifted by $\Delta d_{\mathrm{d}}$ from the real current path, depending on the grid resolution. Additionally the unavoidable discretization errors of the scan grid against the dipole positions $\Delta d_{\mathrm{s}}$, will influence the current estimation. Therefore interpolation methods are applied to achieve the field vectors above each equivalent current. The sum of these discretization errors will influence the results, particularly strong if the solution method has to deal with such kind of ill-conditioned problem.

To create an accurate model, as we mentioned in Rinas et al. (2011), the current paths of a PCB can be achieved from CAD-data of the board, computer tomography or with a high-resolution pre-near-field scan. This means the equivalent currents can be distributed along the known traces. Thus the dipole path shift discretization errors are negligible, the size of the linear map can be reduced and the model becomes more physically.

\section{Results}

In the following section results of the proposed current identification method are presented. It is validated with ideal simulation data first, second with noisy simulation data and later it is applied to real measurement data of a simple test PCB. It consists of a single trace which is fed by an AC voltage source in simulation and a $4 \mathrm{MHz}$ trapezoidal signal in the real setup. The termination is a $100 \Omega$ resistor. Geometry and configuration of the test PCB are shown in Fig. $4 a$ and $b$.

\subsection{Simulation data based results}

\subsubsection{Ideal data}

The magnetic near-field above the test PCB is achieved in a $160 \mathrm{~mm} \times 100 \mathrm{~mm}$ plane $8.5 \mathrm{~mm}$ above ground. The resolution is set to $3.5 \mathrm{~mm}$. Three different models are considered: Model 1, a dipole grid distribution with fixed grid size $(5 \mathrm{~mm})$, without current estimation, solved by least squares method (LSQ); Model 2, a dipole grid distribution with fixed grid size $(5 \mathrm{~mm})$ with current path identification (CPI) solved by regularization method (Reg.); Model 3, a dipole distribution along the real current paths $(5 \mathrm{~mm})$, with current estimation, solved by regularization method.

Figure 5 shows the current amplitude relative errors and phase absolute errors at the specific trace coordinates at a frequency of $100 \mathrm{MHz}$. Figure 6 presents the electric fields at an observation point $\mathrm{P}$ about $1.5 \mathrm{~m}$ far away from the $\mathrm{PCB}$ in a frequency range from $1 \mathrm{MHz}$ to $1 \mathrm{GHz}$. This observation point represents a possible measurement antenna position. It can be seen that the field in horizontal and vertical position is quite accurate for all models from a frequency of about $50 \mathrm{MHz}$ to $1 \mathrm{GHz}$. Below $50 \mathrm{MHz}$ there is a significant deviation for the simple grid model with LSQ method. A good accuracy for current identification in amplitude and phase is only reached by CAD-data based model with regularization. Especially model 1 shows unphysical jumps in current amplitude and phase. At $100 \mathrm{MHz}$ it can be seen, although current distribution does not match the desired and physically correct current, the field is approximated well. When only the field is required for accurate data it seems to be sufficient to find an arbitrary current distribution, which approximates the field in the reference plane.

\subsubsection{Noisy data}

In a next step noise is added to the ideal simulated magnetic near-field. A SNR of $10 \mathrm{~dB}$ is assumed. The same models (Model 1-3) are applied for current identification.

Figure 7 shows the current amplitude relative errors and phase absolute errors at the specific trace coordinates at a 


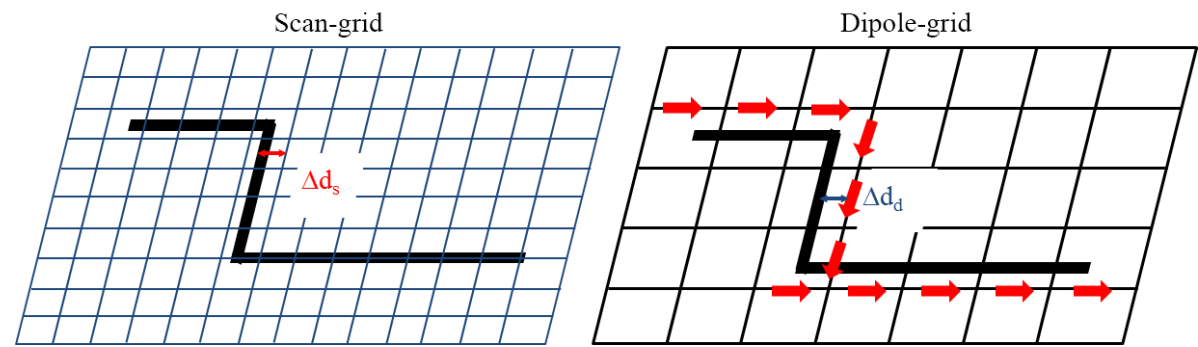

Figure 3. Discretization errors; error in scan plane discretization (left); error in dipole grid discretization (right).

(a)

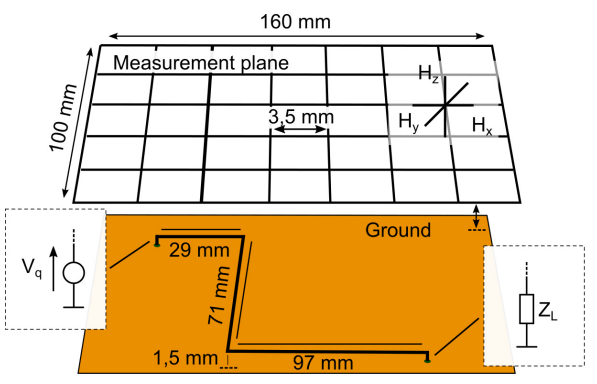

(b)

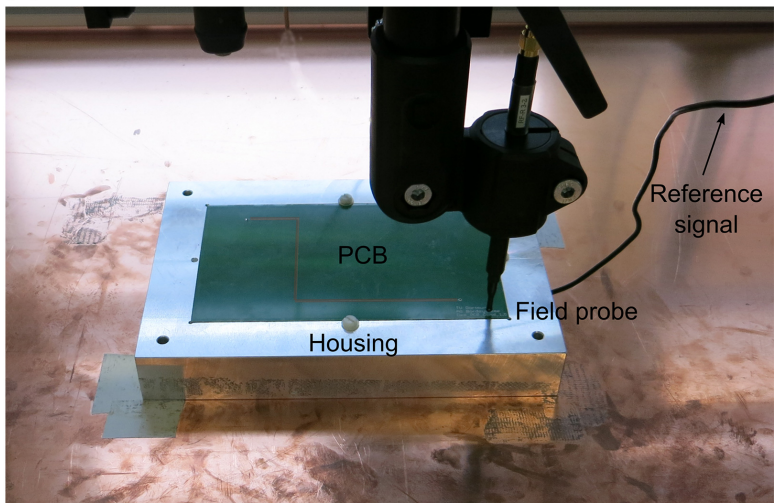

Figure 4. (a) Configuration of test PCB. (b) Picture of PCB scanning.

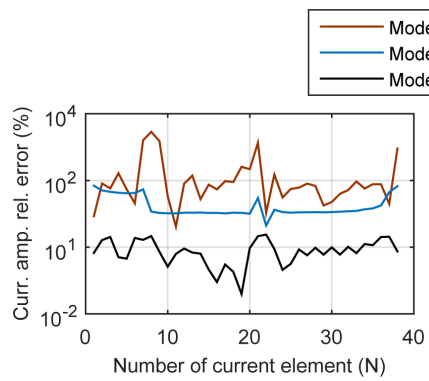

Model 1 (Grid, LSQ)

Model 2 (Grid, CPI, Reg.)

(CAD, CE, Reg.)

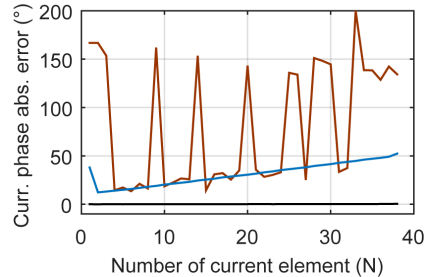

Figure 5. Relative error of current amplitudes (left) and absolute phase error (right) of different models at given trace coordinates $(100 \mathrm{MHz})$

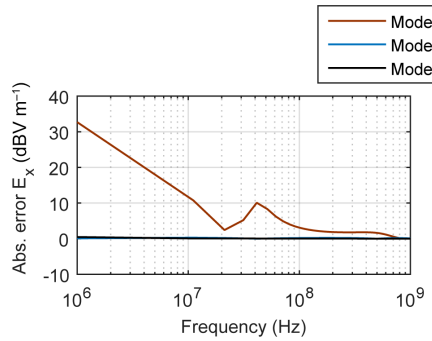

odel 3 (CAD, CE, Reg.)

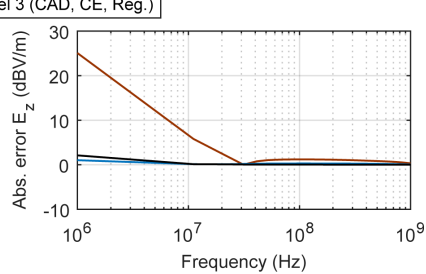

Figure 6. Horizontal (left) and vertical (right) electric field absolute error at observation point $\mathrm{P}$.

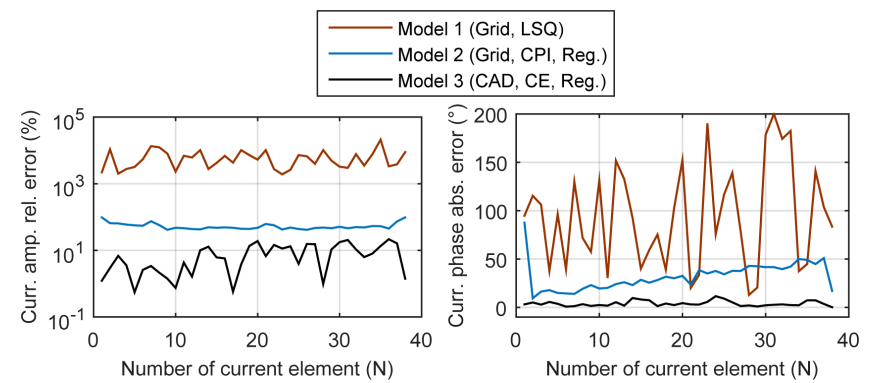

Figure 7. Relative error of current amplitudes (left) and absolute phase error (right) of different models at given trace coordinates $(100 \mathrm{MHz})$.

frequency of $100 \mathrm{MHz}$. Figure 8 presents the vertical component of the magnetic near fields in observation plane. In Fig. 9 the electric fields at an observation point $\mathrm{P}$ about $1.5 \mathrm{~m}$ far away from $\mathrm{PCB}$ in a frequency range from $1 \mathrm{MHz}$ to $1 \mathrm{GHz}$ can be seen. Contrary to the reference data, the model 1 shows a big error in both horizontal and vertical electric field at observation point P. Field calculation based on model 2 results in a much better accuracy in the frequency range of $80 \mathrm{MHz}$ to $1 \mathrm{GHz}$. The CAD-data based approach shows a negligible small deviation starting from a frequency of $20 \mathrm{MHz}$. Again, the current distribution can only be identified sufficiently well with model 3 . The unphysical current 


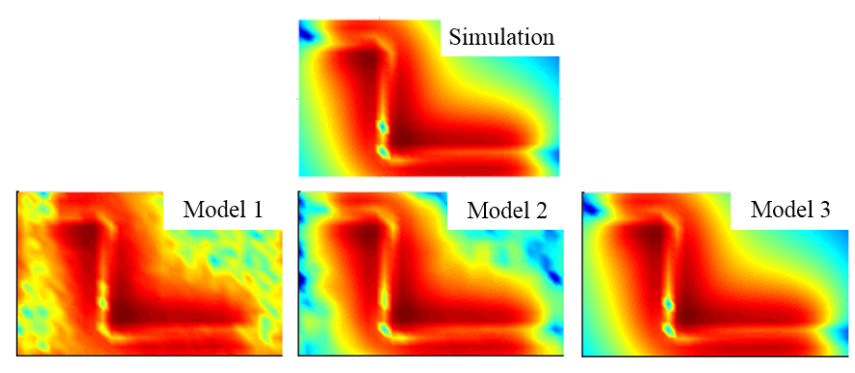

Figure 8. Vertical magnetic near-fields in observation plane compared to the simulated near-field $(100 \mathrm{MHz})$.

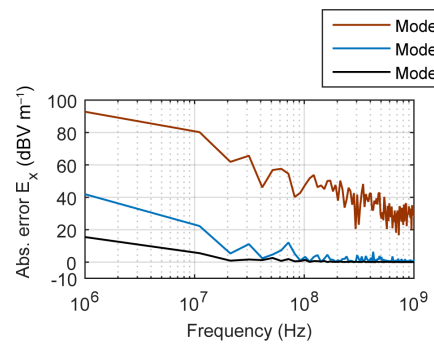

$$
\begin{aligned}
& \text { Model } 1 \text { (Grid, LSQ) } \\
& \text { Model } 2 \text { (Grid, CPI, Reg.) } \\
& \text { Model } 3 \text { (CAD, CE, Reg.) }
\end{aligned}
$$

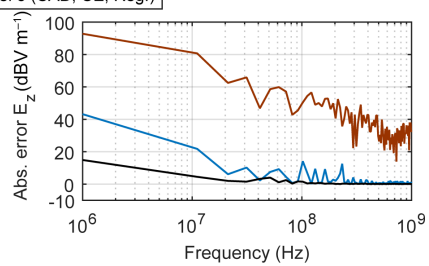

Figure 9. Horizontal (left) and vertical (right) electric field absolute error at observation point $\mathrm{P}$.

is particularly obvious for model 1 which shows current amplitude and phase jumps. For noisy data it is not enough to approximate the field in reference plane to create an accurate model. In Fig. 8 can be seen that regularization and CADdata filter noise and produce a smoothed near field which matches the near field of the undisturbed PCB. This leads to a stable and accurate current and field calculation model.

\subsection{Measurement data based results}

The magnetic field distribution of test PCB is now given by Time Domain measurements (Rinas et al., 2011) with a passive magnetic field probe with $3 \mathrm{~mm}$ loop diameter connected to an oscilloscope. The complex field data is transformed into Frequency Domain with FFT and a reference signal for phase calculation was used. The measurement plane of $160 \mathrm{~mm} \times 100 \mathrm{~mm}$ was located $4.5 \mathrm{~mm}$ above ground.

Figure 10 shows the errors of identified currents for model approach 1 and 3. It can be seen, that the accuracy of the results is improved significantly when using real PCB current trace locations and regularization method.

\section{Conclusions}

Current distribution based radiation models from near field scan data for evaluating the electromagnetic field from PCBs need precise field data for current estimation. As measurement with very high accuracy are often impossible, proposed approaches try to identify the currents using optimiza-

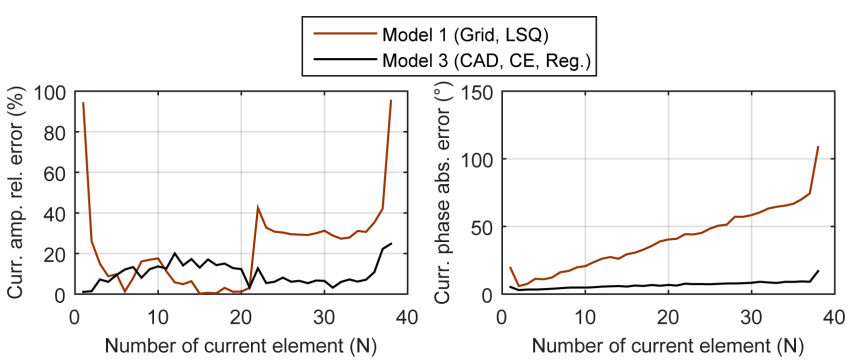

Figure 10. Relative error of current amplitudes (left) and absolute phase error (right) of different models at given trace coordinates $(100 \mathrm{MHz})$.

tion algorithms (e.g. amplitude-only data). Thereby the location, amplitudes and phases can be varied, until one or more desired near-field planes are approximated. Although these methods often find a very good solution for their reference plane, accuracy outside of near-field scan area can be low. Solving the inverse problem with a system of linear equations and complex near-field data can be very sensitive to noise. Regularization methods and an adjusted preconditioning can increase the accuracy. Furthermore, the possible current paths can be restricted to the physical current paths on a PCB. This can be done by CAD-data, computer tomography, or with a high-resolution pre-near-field scan. Additionally the current amplitudes can be estimated from the nearfield data. These precondition measures were implemented for more accurate identification of currents. The benefit and stability of the method was investigated by simulation and measurements with a simple test PCB. The results show an obvious increase of the model accuracy, in the identified currents, and a good error correction in the case of a noisy reference near-field.

\section{Data availability}

Part of this research was done within cooperation projects and is subject to individual confidentiality agreements. Data used for this publication cannot be disclosed.

Edited by: F. Gronwald

Reviewed by: two anonymous referees

\section{References}

Balanis, C. A.: Antenna Theory Analysis \& Design, Wiley, 1996. Burghart, T., Rossmanith, H., and Schubert, G.: Evaluating the RF-emissions of automotive cable harness, International Symposium on Electromagnetic Compatibility, EMC 2004, 3, 787-791, 2004.

CISPR 25 Ed.3: Vehicles, boats and internal combustion engines Radio disturbance characteristics - Limits and methods of measurement for the protection of on-board receivers, IEC, 2007. 
Hanson, R. J.: A Numerical Method for Solving Fredholm Integral Equations of the First Kind Using Singular Values, SIAM J. Numer. Anal., 8, 616-622, 1971.

IEC/TS 61967-6 Ed.1.0: Integrated circuits, Measurement of electromagnetic emissions, $150 \mathrm{kHz}$ to $1 \mathrm{GHz}$ - Part 6: Measurement of conducted emission, magnetic probe method, 2002.

Isernia, T., Leone, G., and Pierri, R.: Radiation pattern evaluation from near-field intensities on planes, IEEE T. Antenn. Propag., 44, 701-710, 1996.

Laurin, J., Zürcher, J. F., and Gardiol, F. E.: Near-field diagnostics of small printed antennas using the equivalent magnetic current approach, IEEE T. Antenn. Propag., 49, 814-828, 2001.

Nishikata, A., Wada, Y., Tawada, M., and Takabe, Y.: Threedimensional dipole source identification using two fixed receiving antennas and its new algorithm, International Symposium on Electromagnetic Compatibility, EMC Tokyo 2014, 33-36, 2014.

Pierri, R., D'Elia, G., and Soldovieri, F.: A two probes scanning phaseless near-field far-field transformation technique, IEEE T. Antenn. Propag., 47, 792-802, 1999.

Regué, J.-R., Ribó, M., Garell, J.-M., and Martin, A.: A Genetic Algorithm Based Method for Source Identification and Far-Field Radiated Emissions Prediction From Near-Field Measurements for PCB Characterization, IEEE T. Electromagn C., 43, 520-530, 2001

Rinas, D., Niedzwiedz, S., Jia, J., and Frei, S.: Optimization methods for equivalent source identification and electromagnetic model creation based on near-field measurements, EMC Europe 2011, 298-303, 2011.
Rinas, D., Zeichner, A., and Frei, S.: Measurement environment influence compensation to reproduce anechoic chamber measurements with near-field scanning, International Symposium on Electromagnetic Compatibility, S. 705-710, 2013.

Sijher, T. S. and Kishk, A. A.: Antenna modeling by infinitesimal dipoles using genetic algorithms, Progress In Electromagnetics, 225-254, 2005.

Tichonov, A. N. and Arsenin, V. J.: Solutions of ill-posed problems, Washington, DC, Winston (A Halsted Press book), 1977.

Xin, T., Thomas, D. W. P., Nothofer, A., Sewell, P., and Christopoulos, C.: A genetic algorithm based method for modeling equivalent emission sources of printed circuits from near-field measurements, Asia-Pacific Symposium on Electromagnetic Compatibility, 293-296, 2010a.

Xin, T., Thomas, D. W. P., Nothofer, A., Sewell, P., and Christopoulos, C.: Modeling Electromagnetic Emissions From Printed Circuit Boards in Closed Environments Using Equivalent Dipoles, IEEE T. Electromagn. C., 52, 462-470, $2010 \mathrm{~b}$.

Yaccarino, R. G. and Samii, Y. R.: Phase-less bi-polar planar nearfield measurements and diagnostics of array antennas, IEEE $\mathrm{T}$ Antenn. Propag., 47, 574-583, 1999.

Yaghjian, A. D.: An overview of near-field antenna measurements, IEEE T. Antenn. Propag., 34, 30-4, 1986.

Zhenwei, Y., Jayong, K., Mix, J. A., Slattery, K., and Jun, F.: Extracting physical IC models using near-field scanning, IEEE International Symposium on Electromagnetic Compatibility, 317320, 2010. 